\title{
Magnetic Resonance Imaging in Malawi: Contributions to Clinical Care, Medical Education and Biomedical Research
}

\section{J Potchen ${ }^{1}$, S. Kampondeni² ${ }^{2}$ G. L Birbeck $^{3}$; C.A'Hammond ${ }^{1}$ A. Gonani ${ }^{2}$, K.S. Phiri $^{4}, K^{3} . B$ Seyde ${ }^{5,6}$; T.E Taylor ${ }^{5,6}$}

1. Michigan State University, Department of Radiology, Radiology 184, East Lansing, MI 48824-USA

2. Queen Elizabeth Central Hospital, Department of Radiology, Chipatala Ave, PO Box 95, Chichiri, Blantyre-MALAWI

3. Michigan State University, International Neurologic \& Psychiatric Epidemiology Program, \#324 West Fee Hall, East Lansing, MI 48824-USA

4. University of Malawi College of Medicine, Dept of Community Health, P/B360, Blantyre 3, Malawi.

5. University of Malawi College of Medicine, Blantyre Malaria Project, Box 32256, Chichiri, Blantyre 3-MALAWI

6. Michigan State University, College of Osteopathic Medicine, Department of Internal Medicine, B309 West Fee, East Lansing, MI 48824-USA

\begin{abstract}
Advanced medical imaging technologies are generally unavailable in low income, tropical settings despite the reality that neurologic disorders are disproportionately common in such environments. Through a series of donations as well as extramural research funding support, an MRI facility opened in Blantyre, Malawi in July 2008. Resulting opportunities for studying common tropical disorders, such as malaria and schistosomiasis, in vivo are promising. The subsequent improvements in local patient care were expected and exceptional and include major revisions in basic care protocols that may eventually impact care protocols at facilities in the region that do not have recourse to MRI. In addition, advanced neuroimaging technology has energized the medical education system, possibly slowing the brain drain. Advanced technologies, though potentially associated with significant fiscal opportunity costs, may bring unexpected and extensive benefits to the healthcare and medical education systems involved.
\end{abstract}

\section{Introduction}

Medical imaging beyond the portable ultrasound remains a luxury in most of sub-Saharan Africa, especially in publicly funded institutions ${ }^{1}$. The initial equipment investment is expensive, as are the running costs, and the technical knowledge required to operate and maintain the equipment is scarce. An auspicious confluence of circumstances resulted in the installation of a $0.35 \mathrm{~T}$ MRI in Blantyre, Malawi in 2008: General Electric Healthcare donated the Signa Ovation Excite (\$1.1 million), the Malawi Ministry of Health made land available on the grounds of the Queen Elizabeth Central Hospital (QECH), the Michigan State University College of Osteopathic Medicine underwrote construction costs for the building housing the MRI $(\$ 150,000)$, and a research grant from the US National Institutes of Health covered the initial running costs $(\$ 100,000 /$ year). The modus operandi of the MRI in Malawi was determined by a steering committee with representatives from QECH, the University of Malawi College of Medicine, and the NIH-funded investigators, and the fundamental principle was that access to the magnet for patients in Malawi government hospitals would be subsidized by research projects and by fees charged to private patients. Prior to the magnet's commissioning in
Malawi, private patients who required imaging would travel to South Africa, incurring transportation and accommodation costs in addition to the fees levied for the imaging and its interpretation, so Malawi-based insurance companies and employer-based health plans supported the local imaging option enthusiastically.

\section{Background}

Over eighty percent of Malawi's 14 million inhabitants reside in rural areas. Healthcare access in the government system is generally via health centers, and when necessary, patients are referred to district hospitals or one of three regional tertiary care hospitals. QECH is one of these, and is also one of two teaching hospitals for the University of Malawi Colleges of Medicine, Nursing and Laboratory Science. Much of the biomedical research in Malawi is carried out in and around QECH. Of the 2,106 patients scanned during the first two years of operation, 237 were research scans and 402 were scans performed for private pay patients; these represented $11 \%$ and $19 \%$ of the total scans performed, respectively.

\section{The MRI}

The scanner is a $0.35 \mathrm{~T}$ permanent resistive magnet and therefore does not require cryogen coolants. Using a permanent magnet, rather than a super-cooled magnet, simplifies the cost and logistical complexity of operations significantly. The GE Signa Ovation Excite has an open configuration which helps to reduce claustrophobia in conscious patients and improves access to unconscious patients, in the event of clinical deterioration. The $0.35 \mathrm{~T}$ GE MRI scanner provides most standard scanning protocols, including T1, T2 FRFSE, EPI-diffusion-weighted imaging, GRE, GRE T2*, proton density, STIR and 2-D and $3-\mathrm{D}$ volumetric imaging. These methods are combined into research and clinical protocols designed for standard anatomic imaging of the brain, head and neck, spinal cord and column, thorax, abdomen, pelvis and extremities. Of note, the relatively low field strength of this scanner has limitations with FLAIR sequences, resulting in poor T2 representation on that image series. T1 image contrast is also low, resulting in limited visualization with gadolinium (MRI contrast agent). MR angiography has relatively poor spatial resolution, making this of limited utility on this scanner.

\section{Impact}

\section{Clinical Care}

The diagnostic information provided by the MRI has enhanced clinical management of patients presenting with a broad range of clinical pathology. The alterations in the management of patients presenting to $\mathrm{QECH}$ with acute or sub-acute, nontraumatic paraplegia that has occurred since MRI became available provide an example of how MRI imaging has positively impacted clinical care. There is a broad differential for a Malawian patient presenting with paraplegia (Table 1). Schistosomiasis, primarily due to Schistosoma haematobium, is endemic in Malawi and is a recognized cause of paraplegia ${ }^{2}$. It is responsible for cord lesions leading to paraplegia and can be well demonstrated using MRI (Fig 1). 
Table 1: Potential Causes of Sub-Acute or Acute, Nontraumatic Paraplegia in Malawi

\begin{tabular}{|c|c|}
\hline Condition & Specific Examples \\
\hline Primary structure failure & $\begin{array}{l}\text { Disc herniation } \\
\text { Spondylolisthesis } \\
\text { Hypertrophic degenerative spurring }\end{array}$ \\
\hline \multirow[t]{4}{*}{ Turnor } & $\begin{array}{l}\text { Schwannoma } \\
\text { Meningioma }\end{array}$ \\
\hline & Burktt's lymphøma \\
\hline & $\begin{array}{l}\text { Metastatic dis ease } \\
\text { Neura fibroma }\end{array}$ \\
\hline & Primary cord tumors \\
\hline Autoimmune & $\begin{array}{l}\text { Myelitis due to mul tiple sclerosis or other } \\
\text { demyelinating disorder }\end{array}$ \\
\hline \multirow[t]{2}{*}{ Vascular } & Dural AV fistul as \\
\hline & $\begin{array}{l}\text { AVM's } \\
\text { Tuberculosis (Pott's'Disease) }\end{array}$ \\
\hline \multirow{2}{*}{ Infectious } & Spinal schistosomiasis \\
\hline & Paraspinal abscess \\
\hline
\end{tabular}

Prior to the introduction of MRI in Malawi, the clinical algorithm used in caring for a patient with acute or subacute, nontraumatic paraplegia consisted of plain X-rays of the spine from 2 directions to identify features suggestive of TB, metastatic malignancy, diminished bone density, vertebral collapse or paraspinal abscess, together with tests for syphilis and HIV, in the context of a full clinical assessment. If a clear diagnosis could be made, treatment for that entity was initiated. If the initial workup was clinically and radiographically nondiagnostic, empiric treatment with praziquantel for presumed schistosomiasis would be started followed by a reassessment after 3 weeks. If symptoms persisted, treatment was directed at the presumed next most common cause for such paraplegia - tuberculosis (TB), which is not evident by conventional radiographs. TB treatment requires six months of multidrug antituberculous therapy. Only after these measures failed was a patient assessed for other treatable causes usually through an exploratory surgery. Such delays frequently resulted in permanent spinal cord injury with high rates of associated morbidity and mortality. Since MRI became available, a new algorithm for care has been established. Acute MRI imaging has afforded a number of patients the opportunity to undergo corrective surgery before permanent spinal cord damage ensues. An example of such a case is shown in Figure 2. The extraosseous extension and mass effect from TB can also be readily identified on MRI (Fig 3) which can greatly enhance surgical planning in clinically indicated cases. This substantive change in the routine clinical paradigm underscores the impact of the MRI on clinical care.

\section{Text Box 1}

The practice of orthopaedic surgery in Malawi has been ransformed MRI. Dr. Nyengo Mkandawire is a frequent user and comments, "The introduction of MRI at QECH has evolutionized the diagnosis and management of orthopaedic and neurosurgical conditions, especially those involving the pine. Since the MRI has been operational we have been ble to diagnose and manage a wide range of conditions ncluding spinal meningioma, lipoma, cryptococcoma, Chiari 1 malformations, syringomyelia, Burkett's lymphoma and of fourse the ubiquitous "TB spine". Without a doubt if the MRI was not here most of these cases would not have been managed appropriately in a timely fashion. Most, if not all have had satisfactory outcomes following surgery thanks to he improved diagnostic capacity made available through the MRI".

Other common CNS conditions in which MRI availability has transformed care include epilepsy due to neurocysticercosis and drainable subdural abscesses due to $\mathrm{S}$ pneumonia.

\section{Medical Education}

Structural, anatomical data made available through patient MRIs greatly enhances the learning experience of medical students and post-graduates and facilitates teaching opportunities for busy faculty. Since the MRI is located adjacent to the teaching hospital, trainees at all levels have the opportunity to review cases either at the console with the interpreting radiologist or in daily teaching rounds. By seeing firsthand how improved imaging accentuates clinical care, it has broadened their view of medicine and their role in it. This has important implications for the practice of medicine in Malawi. "Brain drain" is a significant problem facing Malawi and other countries in the region. Providing an enhanced medical education and clinical practice environment may potentially deter the migration of health professionals from Malawi.

\section{Text Box 2}

Prof. Robin Broadhead, until recently the Principal of the University of Malawi College of Medicine recognizes the contributions of the scanner: "Having the MRI in Malawi has made it possible for us to educate our students more horoughly and creatively. The link between physical findings fnd the underlying anatomy and physiology is crystal clear, Ind that is helpful as the students move out into more remote reas of Malawi. The clinicians have been invigorated by the ncreased precision of their diagnoses - - their enthusiasm is palpable, and makes it easier for me to encourage Malawian loctors abroad to return, and to encourage our graduates to tay at home to practice."

The MRI unit also provides an opportunity for telemedicine. Images and histories are relayed to Michigan State University via DICOM routers where subspecialty medical faculty previously not involved in international work have shown a great willingness to assist through informal consultation. This reduces the professional isolation for Malawi specialists while also improving patient care and offers unique cases for medical education in the US. 
Fig 1: Sagital T2 (A) (FSE TR3200 TE 110 FOV 36) and T1 postcontrast (B) FSE TR 625 TE min full FOV 36) images in a 15 year old male with acute onset paraplegia show an enhancing anterior conus lesion (straight arrows) with extensive cord edema (curved arrows). The focality allowed for surgical resection, and histological analysis of the tissue revealed the presence of Schistosoma haematobium eggs.
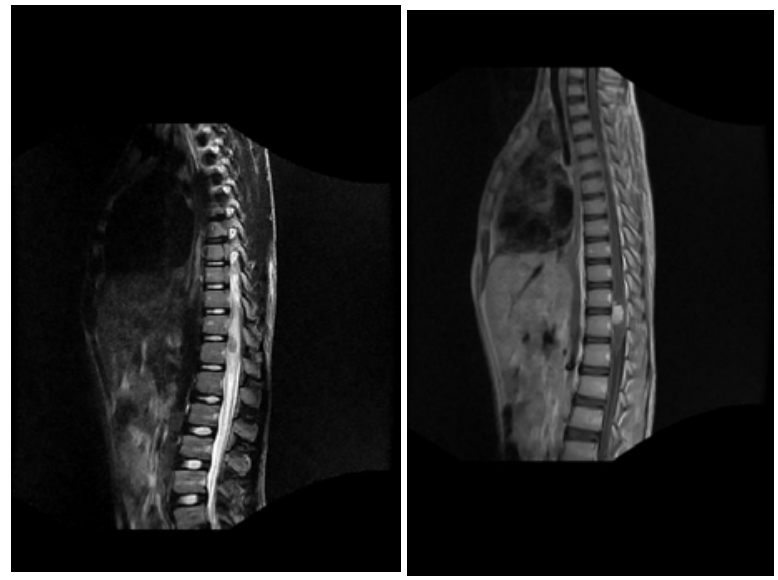

Figure 2: A sagital T2 (A) (FSE TR3200 TE 110 FOV 36) image of the cervical spine in a 14 year old female with progressive subacute quadriparesis. The image shows an anterior; intradural and extramedullary cystic mass with homogenous signal intensity consistent with an enlarging arachnoid cyst. There is associated effacement and compression of the cervical cord, with posterior displacement. This image was the basis for surgical planning and resection which led to a successful neurological outcome.

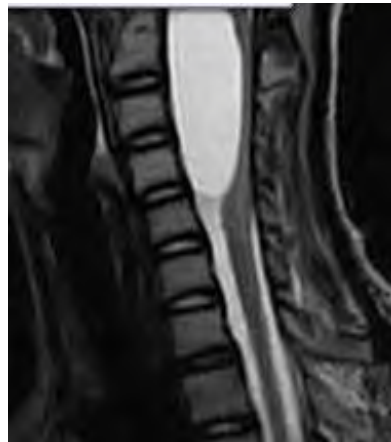

\section{Research}

The primary research activity for the MRI involves adding in vivo imaging data to a long-standing, autopsy-focused study of acute pediatric cerebral malaria. Although the MRI has been operational for $<2$ years, extramural funding for at least two other research projects has already been awarded. One of these is a study of brain MRI findings in pediatric cerebral malaria survivors with neurologic sequelae. This project may offer significant insights into the mechanisms of cerebral malaria-induced epileptogenesis. The population served by the Malawi MRI is exposed to many tropical conditions relatively unique to resource poor regions, such as onchocerciasis, have rarely been imaged before. Data obtained through the Malawi MRI may offer substantial insights into such disorders. Research made possible by the Malawi MRI could eventually offer major advances in our clinicopathologic understanding of tropical diseases and routine patient care.

\section{Challenges}

Numerous challenges emerged during the first year of the MRI operation in Malawi. These included a broad range of scientific, logistical, social, technical and operational issues, many of which are unique to the African setting.
Fig 3: agital T2 (A) (FSE TR3200 TE 110 pFOV 36) and Axial T2 (B) (FSE TR 3800 TE 10828 pFOV) images of a 26 vear old male who presented to Queen Elizabeth Central Hospital (QECH) following acute onset of paraplegia. MRI imaging alfowed for localization and identification of the offending lesion (arrows) as spinal TB, as well as defining its extent, both of which were critical in surgical planning for resection.
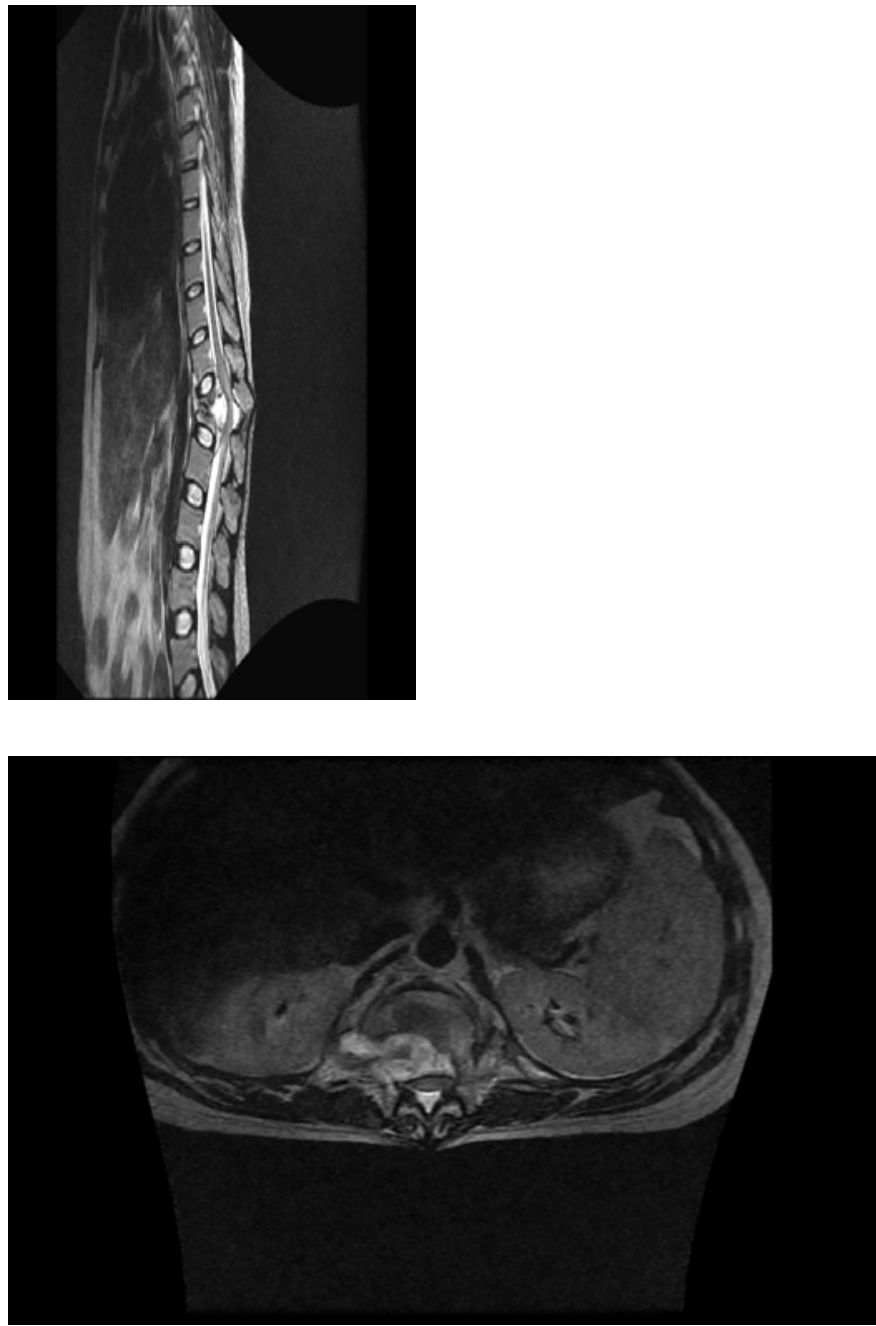

\section{Infrastucture}

An obvious yet important limitation is the capability of a single machine. Even for the population of Blantyre, let alone the whole country, the facility cannot be available for all who need it, and especially not with the promptness that most clinical conditions call for. While it is true that this machine cannot possibly cope with all the needs, its benefits in selected cases may serve as a stimulus to various parties within and outside of Malawi - to aim at increasing diagnostic and investigational modalities in the country.

\section{Resources}

The original model of having research grants and private patients subsidize the imaging of patients in the government health system is working, but will not generate enough "income" to cover the cost of replacing coils, etc.

A natural tension exists between researchers hoping to utilize the MRI for important research relevant to the health of the region and administrators responsible for assuring the longterm sustainability of the MRI. By providing MRI scans free of charge in the acute medical setting, researchers studying acute conditions can obtain study scans without the financial burden of including MRI costs in their research budgets. What constitutes "research" for the purpose of generating income for the scanner? Should studies involving retrospective chart reviews be permitted to use MRI data free of charge? Should authors of individual 'case reports' contribute to the cost of 
running the magnets? Should the grant proposal budgets for prospective studies enrolling patients with a particular clinical condition include the real cost of the MRIs? Such questions illustrate the complexities of maintaining ongoing fiscal solvency for this resource.

Similarly, the private sector provides a complex microcosm of the societal issues related to the long term sustainability of the MRI. One such example is the dilemma which arises out of the patients in the private sector who do not qualify for, but who routinely seek, care in the public sector. Already, private patients and their physicians have tried various approaches to circumventing payment schemes, and although steps are being taken to reduce the budgetary impact, loopholes such as these produce challenges for the long-term sustainability of the MRI enterprise.

\section{Interpreting the findings}

Shortly after imaging of acutely ill patients began, numerous pathological findings were identified which either appeared chronic or had no apparent correlation with the acute clinical presentation. Specifically,

1). Brain MRIs obtained for acute clinical illness exhibited structural abnormalities more likely to be associated with a pre-existing injury and/or chronic neurologic problem (e.g. significant generalized or focal cortical atrophy). (Fig 4)

2). Structural abnormalities were seen which are not welldescribed in existing autopsy or MRI literature. These include unusual unilateral hemispheric atrophy in pediatric populations (Fig 5)

3). Brain MRIs of acutely ill patients showed findings of unclear significance, such as subclinical hydrocephalus.

Interpreting brain MRI studies obtained in Malawi has proven at times to be extremely difficult. Populations differ in their exposure to a series of potential CNS insults including antenatal trauma, recurrent infections, parasitic infestation, and physical deprivation. These exposures might certainly result in neuro-anatomical abnormalities evident on MRI images. Unfortunately, without normative data regarding the underlying rate and clinical significance of these structural abnormalities within the Malawian population, it is difficult to place abnormal CNS imaging findings in their appropriate context. The potential for falsely attributing coincidental findings to clinical conditions of interest is substantial. Indeed MRI technology has the potential to contribute more confusion than clarification to clinical neurosciences in the tropics.

Acquisition of normative data is important in the evolution of any new technology as it develops and its utility is disseminated. After MRI technology originally became available in the US, a number of systematic studies to outline the range of normal findings and variations were conducted ${ }^{3-7}$.Importantly, formal studies systematically conducted for normative data acquisition complemented the experience of radiologists who, through reviewing numerous clinical studies acquired in relatively healthy individuals, developed a clinical sense of the range of "normal" and an appreciation of the evolution of various anomalies seen ${ }^{3,5}$. But such knowledge is predicated on imaging people with a range of illness severity and the availability of serial images within the same individual. The understanding that without formal, deliberate and prospective studies to acquire normative imaging data in this population has led to another $\mathrm{NIH}$ funded research project aimed at directly addressing this

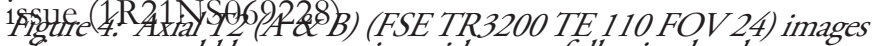
of a one year old boy presenting with coma following head trauma shows the presents of diffuse cerebral atrophy which predates the acute event.
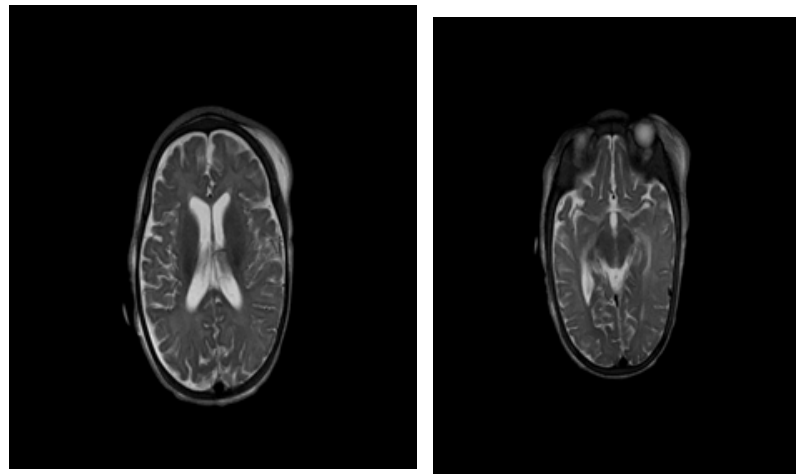

Figure 5: Axial T2 (A \& B) (FSETR3200 TE 110 FOV 24) and coronal T2 (C) (FSE TR 3200 TE 110 FOV 110) images of a three year old boy presenting to the paediatric research ward in coma due to cerebral malaria show unilateral left hemispheric atrophy. This is an example of chronic structural abnormalities seen during the acute imaging which is not well-described in existing autopsy or MRI literature. Hemiatrophy has been identified in $4.1 \%$ of acute cerebral malaria cases imaged to date.
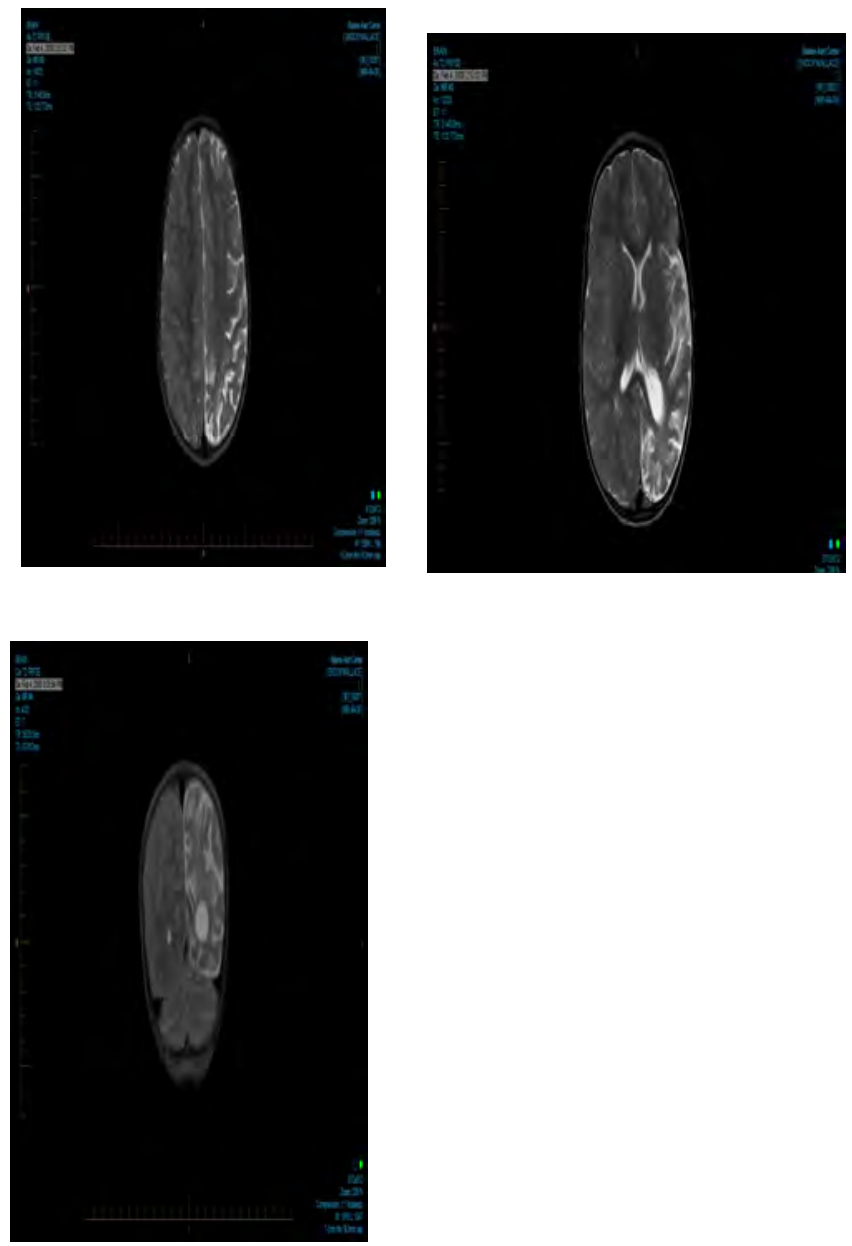

\section{Conclusions}

MRI technology provides enhanced diagnostic options for the people of Malawi, improves medical education, and affords a rare opportunity for clinical and basic science researchers to utilize advanced imaging for medical research expanding our understanding of neglected and under-studied conditions unique to tropical, resource poor regions. This MRI 
facility complements existing capacities at QECH in clinical neurophysiology, clinical neuropsychiatry, ophthalmology (including fundus photography and fluorescein angiography) and basic laboratory assessments (microbiology, clinical chemistry, and clinical hematology). This rare combination of advanced technologies opens up new opportunities for research on topics for which resources have previously been insufficient.

\section{Acknowledgments}

We would like to acknowledge Cowles Chilungulo for thoughtful technical assistance, Dina Kayaye for administrative support, Esther Gondwe for wise oversight and coordination during construction and implementation, John Valentine for project management during construction and installation, and Eric Borgstein for representing the College of Medicine during the preparatory phases of this project. Appreciation also to General Electric, Dean William Strampel, and Professor E James Potchen who collaborated to make the MRI Facility a reality.

\section{Bibliography}

1. WHO/WFN. Atlas: Country resources for neurological disorders. Geneva: WHO; 2004.

2. Carod-Artal FJ. Neuroschistosomiasis. Expert Rev Anti Infect Ther. Nov;8(11):1307-18.
3. Katzman GL, Dagher AP, Patronas NJ. Incidental findings on brain magnetic resonance imaging from 1000 asymptomatic volunteers. Jama. 1999 Jul 7;282(1):36-9.

4. Kim BS, Illes J, Kaplan RT, Reiss A, Atlas SW. Incidental findings on pediatric MR images of the brain. AJNR Am J Neuroradiol. 2002 Nov-Dec;23(10):1674-7.

5. Kleinschmidt A. Incidental neuroimaging findings: lessons from brain research in volunteers. Curr Opin Neurol. 2007 Aug;20(4):387-9.

6. Tsushima Y, Taketomi-Takahashi A, Endo K. Prevalence of abnormal findings on brain magnetic resonance (MR) examinations in adult participants of brain docking. BMC Neurol. 2005;5:18.

7. Weber F, Knopf H. Incidental findings in magnetic resonance imaging of the brains of healthy young men. J Neurol Sci. 2006 Jan 15;240(12):81-4. 